\section{Reduction of Knee Pain by Effusion Reduction}

To the Editor:

The article in The Journal by Elsaman, et al ${ }^{1}$ is quite thought-provoking. If elimination or reduction of knee effusion is the mechanism of action of spironolactone in reducing knee pain ${ }^{1,2}$, the implication is that the effusion is not of an inflammatory nature but rather that it is mechanically derived. It further suggests that structural damage does not fully explain the pain noted in patients with osteoarthritis of the knee, independent of severity. It has been hypothesized that a significant component of the pain is derived from joint instability related to damage to fibular and tibial collateral knee ligaments. A commentary ${ }^{3}$ (in press) noted the effectiveness in relieving knee pain of a specific exercise program in which strengthening the quadriceps mechanism compensates for the ligamentous laxity. While it had been assumed that the efficacy of that approach related simply to reduction of instability, perhaps the mechanism of that intervention may also be related to effusion reduction, as implied by the observations of Elsaman, et $a l^{1}$.
BRUCE ROTHSCHILD, MD, Professor of Medicine, Northeast Ohio Medical University. Address correspondence to Dr. B. Rothschild, Department of Medicine, Northeast Ohio Medical University, Rootstown, Ohio 44272, USA. E-mail: spondylair@gmail.com

\section{REFERENCES}

1. Elsaman AM, Radwan AR, Mohammed WI, Ohrndorf S. Low-dose spironolactone: treatment for osteoarthritis-related knee effusion. A prospective clinical and sonographic-based study. J Rheumatol 2016;43:1114-20.

2. Paschos NK, Giotis D, Abuhemond K, Georgoulis AD. Effectiveness of aspiration in knee joint effusion management: A prospective randomized controlled study. Knee Surg Sports Traumatol Arthrosc 2014:22:226-32.

3. Rothschild BM. Primary or secondary effect of quadriceps exercises on subsequent need for knee replacement. Arthritis Rheum (in press).

J Rheumatol 2016;43:12; doi:10.3899/jrheum.160790 